\title{
Successive Schwarzschild spheres and other rigidity frontiers in spherically symmetric dust-plus-vacuum spacetimes
}

\author{
J. Ehlers \\ Max-Planck-Institut für Astrophysik, Karl-Schwarzschild-Strasse 1, W-8046 Garching, Germany
}

and

W. Rindler

Physics Department, The University of Texas at Dallas, Richardson, TX 75083-0688, USA

Received I June 1993; accepted for publication 16 July 1993

Communicated by J.P. Vigier

\begin{abstract}
Just outside a Schwarzschild sphere concentric rigid test matter spheres can persist while just inside they cannot. We construct spacetimes containing successive Schwarzschild spheres and show that there exist similar but moving rigidity frontiers between them. Some of our solutions exemplify inextensible nonempty spacetimes with Kruskal-like topology having two asymptotically flat ends of possibly different Schwarzschild ( $\equiv$ ADM) mass.
\end{abstract}

\section{A paradox: rigidity frontier defined}

Consider a Schwarzschild black hole, surrounded by vacuum and, at a sufficiently large distance, by a concentric and spherically symmetric infalling shell of dust of finite thickness. Outside the shell there is vacuum again. If the shell spawns its own "Schwarzschild sphere" (a sphere whose history is a null hypersurface and which maintains a constant area ) off its outer surface, is there not then a paradox? For just inside the outer Schwarzschild sphere a particle cannot remain at rest at constant areal radius $l$ (making the area of a sphere $l=$ const equal to $4 \pi l^{2}$ ), while just outside the inner one it can! The paradox is resolved by the recognition of a spherical locus travelling inwards within the shell from the outer to the inner Schwarzschild sphere, and separating the regions where particles can or cannot satisfy $l, \vartheta, \phi=$ const. We call such a locus a rigidity frontier: to one side of it a concentric "rigid sphere" $l=$ const can exist while to the other side it cannot. (Rigid spheres may here be thought of as lattices constructed of light rods of constant proper length, the spheres thus having constant area.)
A trivial example of a rigidity frontier, as we have noted, is the Schwarzschild sphere $l=2 m$ of a Schwarzschild black hole of mass $m$. But neither its nullity (i.e. being a potential light front) nor its being of constant area are properties necessarily shared by the general rigidity frontier. For example, in the case of a collapsing Friedman universe, the rigidity frontier is swept in towards the singularity by the infalling matter at sub- or superluminal speed, as we show in section 2.

Such loci have been recognized before under somewhat different guises. In his classic paper on spherically symmetric dust spacetimes [1], Bondi discovered the possibility of a "barrier to [radially] outward-moving rays of light" in a spherically collapsing cloud of dust. Among others, Christodoulou [2] again noted this barrier as "the locus of turning points of the outgoing light rays", and identified it with the apparent horizon. The latter - in slight extension of Hawking's original definition [3] - is nowadays taken to be the entire boundary of the region of trapped surfaces. The equivalence of rigidity frontier, light-turn-around locus and apparent horizon in spherically symmetric spacetimes (in more 
general spacetimes only the apparent horizon is even defined) stems from their common four-dimensional characterization: They all are the locus $\Sigma$ of events at which the squared normal

$L=g^{\alpha \beta} l_{, \alpha} l_{, \beta}$

of the hypercylinders of constant areal radius $l$ vanishes, i.e. at which the light-cones touch these cylinders. (See, for example, fig. 1.) On one side of $\Sigma$, $L$ is positive and the cylinders $l=$ const have no intersection with the light-cones with vertex on them, while on the other side of $\Sigma, L$ is negative and the light-cones intersect $l=$ const. We note that for Schwarzschild spheres the light-turn-around property degenerates into light just staying in $\Sigma$.

\section{Rigidity frontier in Friedman big-crunch universes}

By way of example, and for later use in this paper, we shall examine the rigidity frontiers in collapsing Friedman (homogeneous-isotropic-dust) universes. Any such universe can be described by a metric of the form (cf. ref. [4], eq. (9.26))

$\mathrm{d} s^{2}=\mathrm{d} t^{2}-R^{2}(t)\left(\frac{\mathrm{d} r^{2}}{1-k r^{2}}+r^{2}\left(\mathrm{~d} \vartheta^{2}+\sin ^{2} \vartheta \mathrm{d} \phi^{2}\right)\right)$,

with a time range $t_{\text {initial }}<t<0$ and an expansion factor $R(t)$ that tends to zero with $t$. The curvature index $k$ can be \pm 1 or 0 and the comoving radial coordinate $r$ has the range $0 \leqslant r<\infty$ unless $k=1$, when it has a maximum $r=1$ on the cosmic equator (a coordinate singularity). As is customary, we employ units making $c=G=1$.

The areal radius $l$ at time $t$ is here given by

$l=R(t) r$

and so, by reference to (1) and (2),

$L=r^{2}\left(\dot{R}^{2}+k\right)-1$,

where the overdot denotes $\mathrm{d} / \mathrm{d} t$. Consequently, if the factor $\dot{R}^{2}+k$ is positive, a rigidity frontier $(L=0)$ exists and is given by

$r=\left(\dot{R}^{2}+k\right)^{-1 / 2}$.

But a Friedman universe satisfies the Einstein field equations for dust, and these now read (see, for example, ref. [4], eqs. (9.75) and (9.76))

$\dot{R}^{2}+k=C / R+\frac{1}{3} A R^{2}$,

with

$C=\frac{8}{3} \pi \rho R^{3}$.

Equation (6) shows that for a nonnegative cosmological constant $A, \dot{R}^{2}+k$ will be positive and then a rigidity frontier exists for the entire duration of a model's existence. When $A$ is negative, we necessar. ily have an "oscillating" universe (see ref. [4], fig. 9.9), in which $\dot{R}=0$ characterizes the moment $t=\hat{t}$ of maximal extension. Near either end of such a universe $R \rightarrow 0$, the $C$-term dominates the r.h.s. of eq. (6), and a rigidity frontier exists. But if $k=-1$, there will be a whole period around $\hat{t}$ when no such frontier exists, while if $k=0$, the rigidity frontier only momentarily goes to infinity at $\hat{t}$.

For a point riding radially with the locus (5) it is easily verified that

$\mathrm{d} s^{2}=\mathrm{d} t^{2}\left(1-\frac{R^{2} \ddot{R}^{2}}{\left(\dot{R}^{2}+k\right)^{2}}\right)$,

and the expression in large parentheses can be positive or zero or negative. Near a big crunch it is always $\sim \frac{3}{4}$, as can be verified by noting from (6) that $R \rightarrow 0$ implies $\dot{R}^{2} \sim C / R$ and thus $R \sim$ const $\times t^{2 / 3}$. It follows that the rigidity frontier comes into the big crunch with subluminal speed (in fact with equation $\left.l \sim \frac{3}{2}|t|\right)$. But it can also move superluminally: for example, near the moment of maximal extension ( $\dot{R}=0)$ of a flat $(k=0)$ "oscillating" universe the expression in large parentheses clearly becomes negative. Figure 1 shows a Friedman big-crunch model with its rigidity frontier.

The rigidity frontier in its rôle as light-turn-around locus throws some light on the often asked question to what extent an entire universe collapsing to a big crunch resembles a star that has collapsed through its Schwarzschild sphere to form a black hole. While the actual light-turn-around locus lies inside the collapsing star (we shall see this in the next section) its surface takes over that rôle for light emitted from the surface, which immediately falls inwards, in terms of $l$. In a collapsing universe, on the other hand, the light emitted by any one of its fundamental particles (galaxies) is confined to ever smaller spheres $l=$ const 


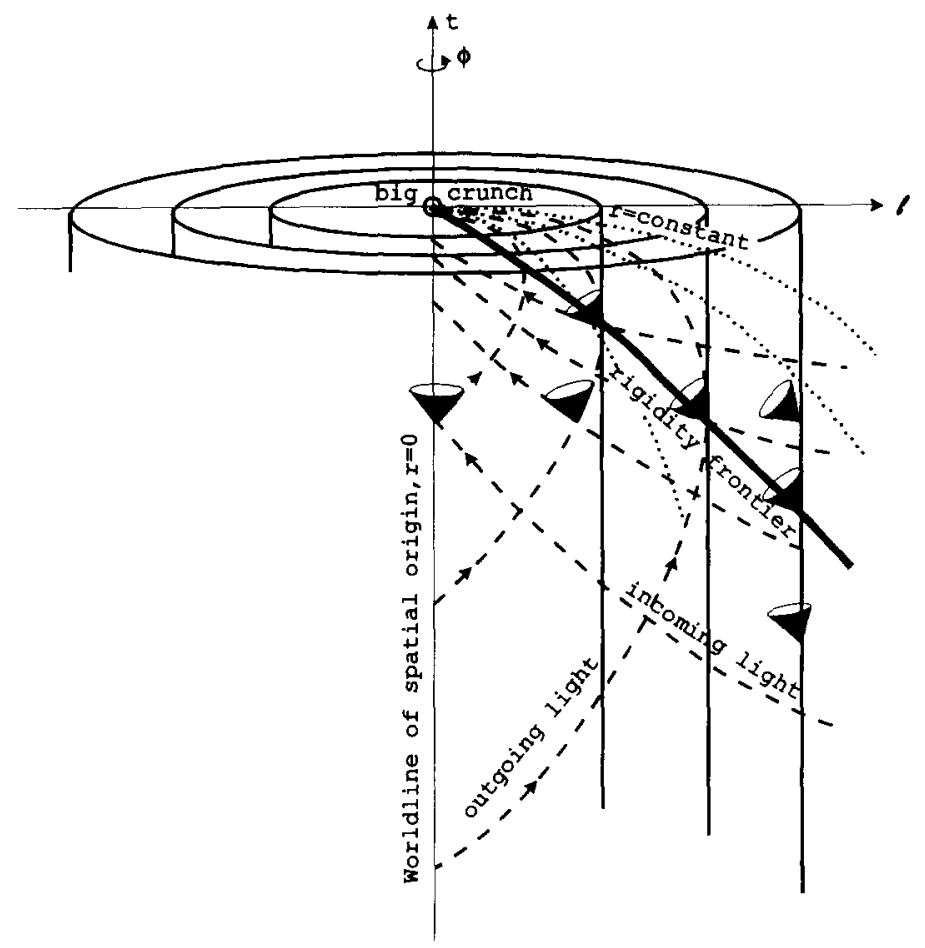

Fig. 1. A collapsing Friedman universe, showing radially incoming and outgoing rays of light. Note how the lines $l, \phi$ (and $\theta$ ) $=$ const touch the light-cones originating on them along the rigidity frontier, but lie inside (outside) these cones inside (outside) the frontier.

around that particle, but within these spheres the light rises first and then falls.

\section{Kruskal-like and other dust-plus-vacuum solutions with successive Schwarzschild spheres}

We are now ready to construct spacetimes with successive Schwarzschild spheres of the kind envisaged in the "paradoxical" example of our introduction. To begin with, let us remove all the matter beyond the comoving sphere $r=r_{0}$ of some collapsing Friedman universe. It is well known [5] that such removal does not affect the motion of the remaining dust ball, and that the junction conditions between the ball and the outside vacuum field can be satisfied by taking for the latter a Schwarzschild metric with suitable parameter $m$, say $m=m_{0}$. Since the outside metric then has its rigidity frontier at $l=2 m_{0}$, the collapsing ball spawns its Schwarzschild sphere at a cosmic time $t_{0}$ when its rigidity frontier coincides with that of the outside vacuum, i.e. from (3) and (5) when

$R\left(t_{0}\right)\left[\dot{R}^{2}\left(t_{0}\right)+k\right]^{-1 / 2}=2 m_{0}$.

In conjunction with (6) and (7), where we now assume $A=0$, this gives

$m_{0}=\frac{1}{2}\left(\frac{8}{3} \pi \rho_{0}\right)^{-1 / 2}$,

$\rho_{0}$ being the density of the dust at time $t_{0}$. This Schwarzschild ( $\equiv$ ADM) mass of the collapsing ball agrees with the naive Euclidean value $\frac{4}{3} \pi l^{3} \rho_{0}$ with $l=2 m_{0}$, even though in general the geometry inside the ball is not Euclidean and its kinetic and potential energies contribute to its total mass.

Our above calculations also make it evident that the rigidity frontier of a collapsing Friedman universe is momentarily at the precise location where the Schwarzschild horizon of the included matter would be if the exterior matter were instantaneously replaced with vacuum.

Our next step is to remove an inner ball bounded 
by a comoving sphere $r=r_{1}<r_{0}$, and to "fool" the remaining shell into moving as if nothing had happened, by replacing the missing dust with a concentric black hole of suitable mass $m_{1}$. This could be either an "already collapsed" ball of dust (as in fig. 2 ) or, alternatively, a Kruskal vacuum black hole complete with wormhole and a second Schwarzschild space of the "far side". (Figure 3 shows this situation for a shell that has been cut from an entire oscillating Friedman universe.) The mass $m_{1}$ we expect to be given by a formula analogous to (9),

$m_{1}=\frac{1}{2} R\left(t_{1}\right)\left[\dot{R}^{2}\left(t_{1}\right)+k\right]^{-1 / 2}$,

$t_{1}$ now being the time when the inner edge of the shell meets the Schwarzschild sphere at $l=2 m_{1}$. Indeed, using once more the methods of ref. [5] it can be verified that with (11) the junction conditions be- tween the shell and the inner vacuum solution are satisfied.

The rigidity frontier inside the shell comes into existence when the event horizon emerges from the outer boundary of the shell. Thereupon it travels within the shell from $r_{0}$ to $r_{1}$. It reaches $r_{1}$ precisely at the moment when the inner boundary of the shell hits the inner Schwarzschild sphere, for at that moment it would pass into the now missing central dust ball. From then into the future no internal rigidity frontier exists and all rigid (particle or photon) spheres become impossible inside the outer Schwarzschild sphere. In particular, the inner Schwarzschild sphere (a potential "outgoing" photon sphere), which up to this instant had "stood still" at $l=2 m_{1}$, is now itself swept into the singularity.

As illustrated by fig. 3 , the two Schwarzschild ends

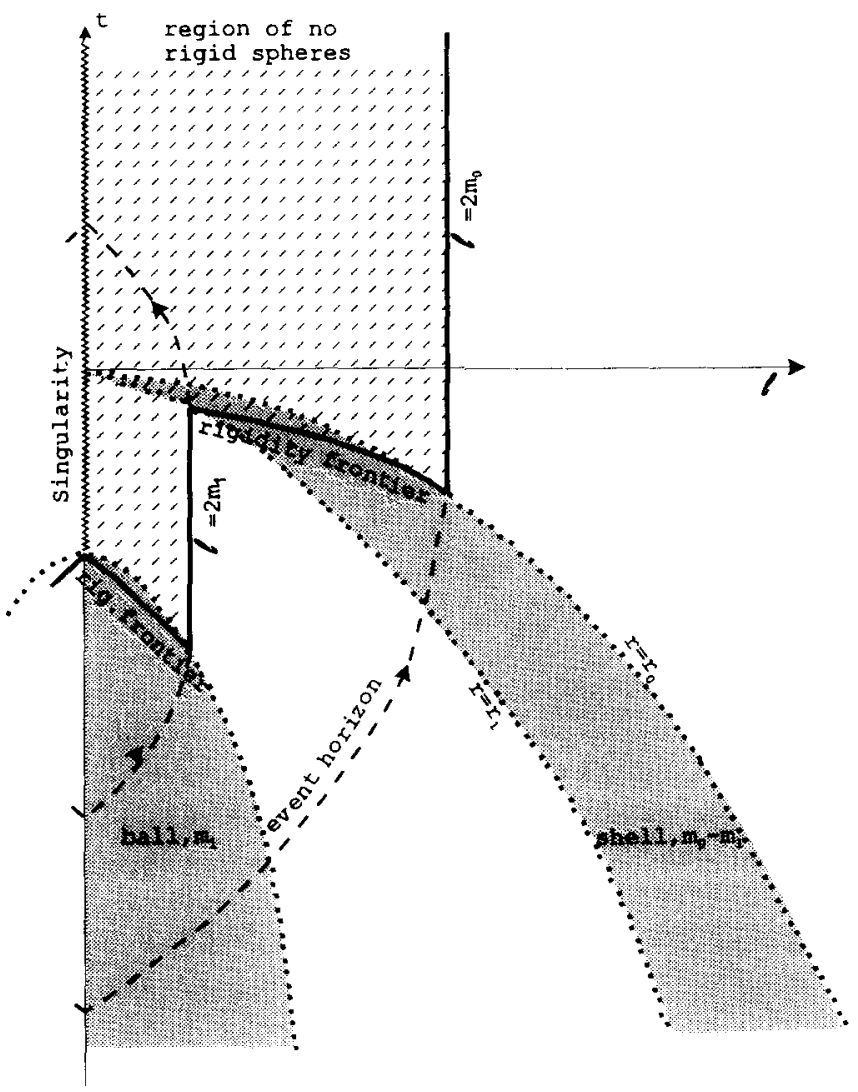

Fig. 2. A collapsing ball of dust surrounded by vacuum and a concentric collapsing shell of dust which is part of the universe of fig. 1. The (stippled) region where no rigid spheres can persist is bounded by the Schwarzschild spheres $l=2 m_{0}, l=2 m_{1}$ and by the rigidity frontiers inside the dust. 


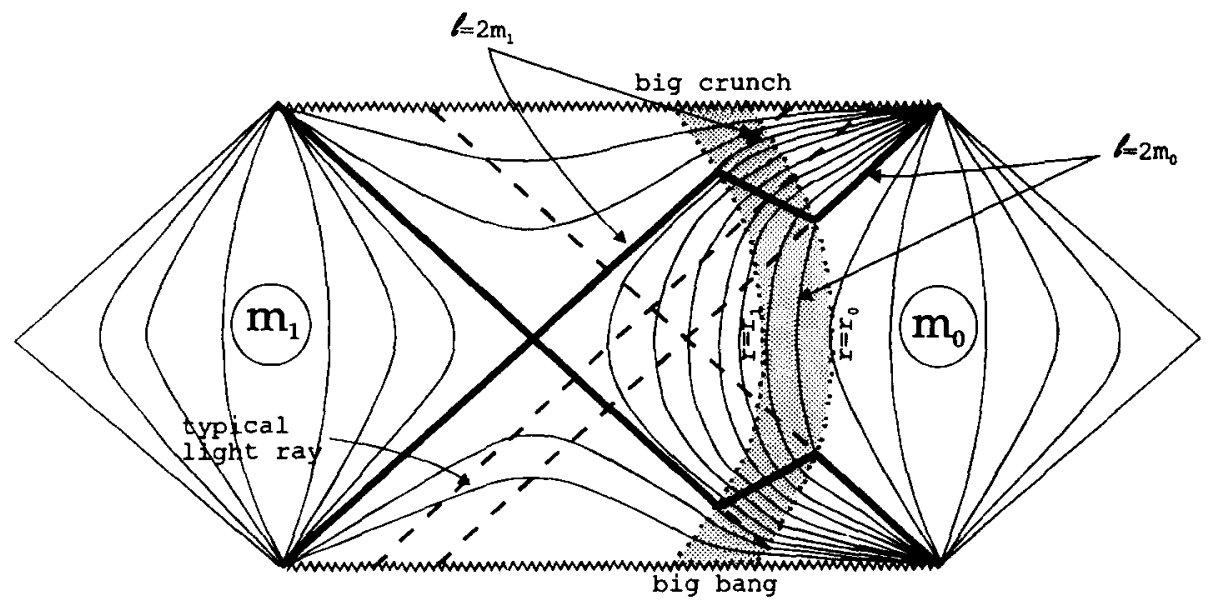

Fig. 3. A Penrose conformal diagram of a zone of an oscillating Friedman universe which is continued both inside and outside by suitable portions of Kruskal vacuum spacetimes. All $\pm 45^{\circ}$ lines are potential light paths. The lines $l($ and $\phi, \theta)=$ const have variable slope here; only where they are steeper than $\pm 45^{\circ}$ are rigid spheres possible.

of the Kruskal-like solutions we here constructed will generally have different masses. Of course, these masses cannot be ascribed directly to the shell, since they are at least partly caused by the vacuum wormhole (Wheeler's "mass without mass") and would simply equalize if the shell were to become infinitely thin.

An interesting variation of the above shell solution can be obtained by considering shells with two interiors, into both of which a Kruskal wormhole leading to a permanently undisturbed outer Schwarz- schild space can be fitted. We need merely start with a closed $(k=1)$ oscillating Friedman universe and cut from it a shell containing the equatorial sphere $r=1$. Figure 4 is a conformal diagram for such a solution in the symmetric case where both surfaces of the shell have the same area. Figure 5 shows a typical constant-time section of this solution, with one spatial dimension suppressed (circles represent spheres). Note the swollen Kruskal neck containing the dust shell between two Schwarzschild spheres, all within another Kruskal neck that joins two permanently un-

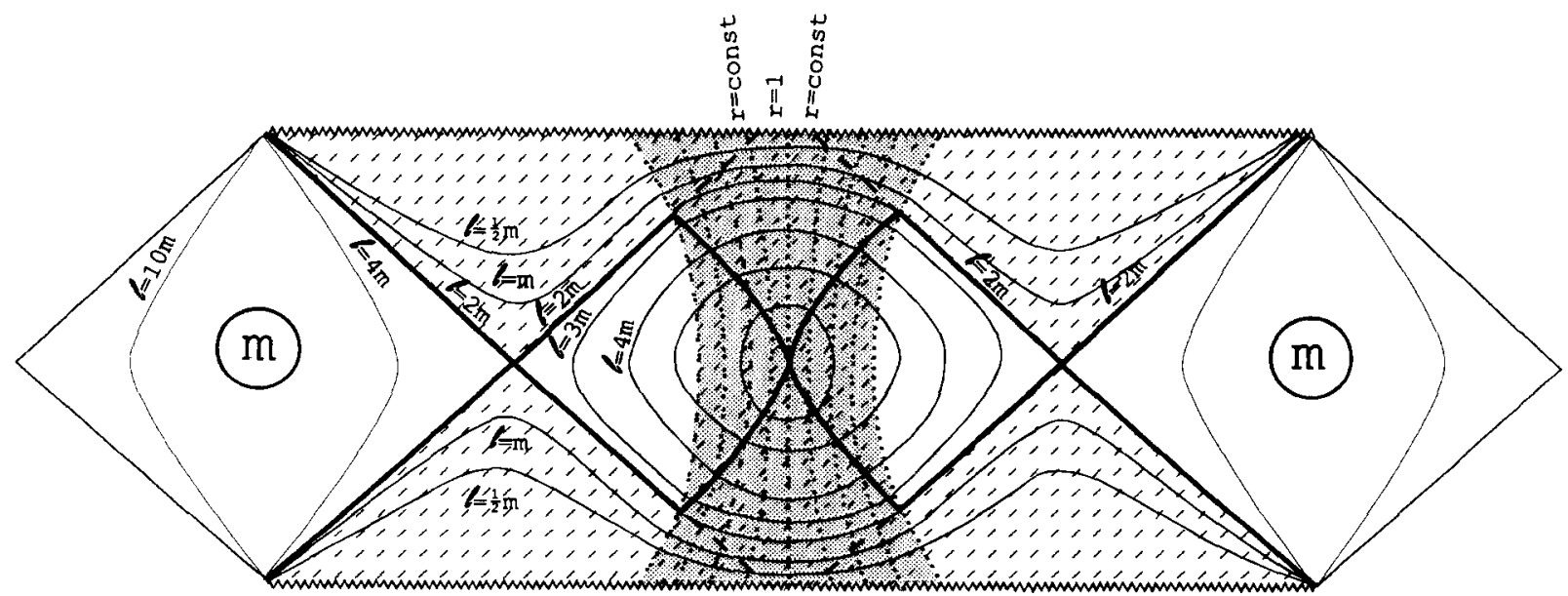

Fig. 4. A variation of the situation of fig. 3: the zone now includes (and is symmetric about) the equatorial sphere $r=1$. Thus it has two "insides", both of which are here joined to Kruskal wormholes leading to undisturbed outer Schwarzschild spacetimes. 


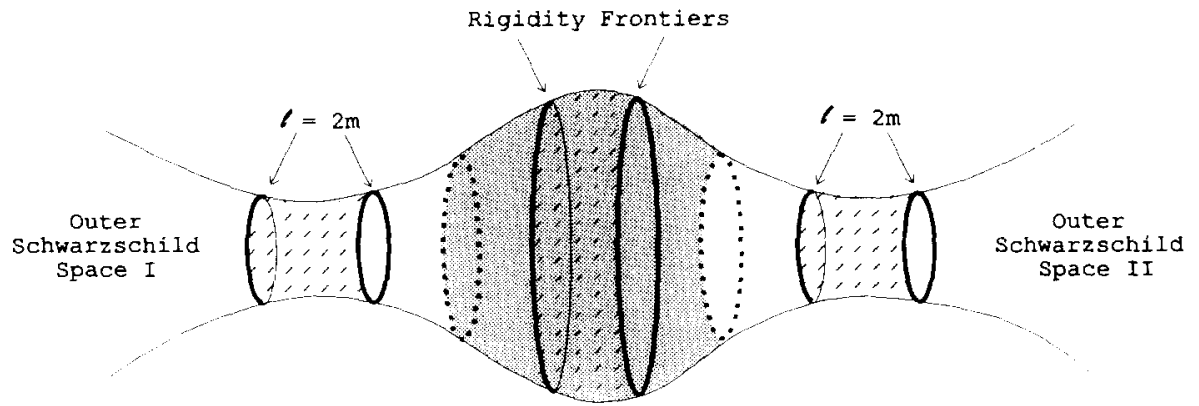

Fig. 5. A typical spatial (horizontal) section through fig. 4, with one dimension suppressed: circles represent spheres. The two outer Schwarzschild spheres $l=2 m$ ultimately go to infinity, while the two inner ones ultimately get absorbed by the dust, whereupon the entire wormbole shrinks to a line.

disturbed outer Schwarzschild spaces.

The results obtained here for simple Friedman shells should be representative of what happens in the case of more general collapsing spherically symmetric fluid shells, e.g. shells lacking homogeneity and possibly having nonvanishing stresses. The situation can also be generalized by having further fluid shells of sufficient density to create any number of consecutive Schwarzschild spheres with - undoubtedly - rigidity frontiers travelling between them.

Künzle in 1967 [6] raised the question whether the field equations of general relativity permit complete singularity-free spherically symmetric spacetimes containing an exterior Schwarzschild region and a massive body but no center. He constructed static configurations of this kind with nonnegative density and anisotropic stresses and established that somewhere inside the body the radial tension has to exceed the density, thus violating the weak, strong and dominant energy conditions. However, if we drop Künzle's staticity ansatz, and ask for inextensible rather than complete spacetimes, then our above solutions in which shells cut from oscillating Friedman universes are joined at both sides to suitable portions of Kruskal spaces, provide examples of the spacetimes in question. And since they contain only dust and vacuum, they trivially satisfy all energy conditions. A more general method for constructing Kruskal-like dust-plus-vacuum solutions has been given by Hellaby [7], and infinitely thin shells of massless matter (null dust) with Kruskal-like spacetimes have been constructed by Dray and 't Hooft [8].

\section{Acknowledgement}

We greatly appreciate early conversations with Jack Denur which led us to invent and resolve the "paradox". It was he who intuited the existence of a horizon-like sphere inside matter whose mass content would just suffice to form a Schwarzschild horizon if the outside were vacuum.

\section{References}

[1] H. Bondi, Mon. Not. R. Astron. Soc. 107 (1947) 410.

[2] D. Christodoulou, Commun. Math. Phys. 93 (1984) 171.

[3] S.W. Hawking, in: Black holes, eds. C. De Witt and B.S. De Witt (Gordon and Breach, New York, 1973) p. 9;

S.W. Hawking and G.F.R. Ellis, The large scale structure of space-time (Cambridge Univ. Press, Cambridge, 1973) p. 320.

[4] W. Rindler, Essential relativity, 2nd Ed. (Springer, Berlin, 1977).

[5] C.W. Misner, K.S. Thorne and J.A. Wheeler, Gravitation (Freeman, San Francisco, 1973) p. 853, ex. 32.4.

[6] H.P. Künzle, Proc. R. Soc. A 297 (1967) 244.

[7] C. Hellaby, Class. Quantum Grav. 4 (1987) 635.

[8] T. Dray and G. 't Hooft, Commun. Math. Phys. 99 (1985) 613. 